\title{
Electrically Isolated Sensor Networks Using Single Polymer Fibers for Remote Powering and Data Transmission
}

\author{
J. Fischer ${ }^{1}$, T. Schuster ${ }^{1}$, C. Wächter ${ }^{1}$, M. Luber ${ }^{1}$, J. Vinogradov ${ }^{1}$, R. Engelbrecht ${ }^{1}$, O. Ziemann ${ }^{1}$ \\ 1 Technische Hochschule Nürnberg Georg Simon Ohm - Polymer Optical Fiber Application Center \\ (POF-AC), Wassertorstr. 10, 90489 Nürnberg, Germany \\ jakob.fischer@pofac.th-nuernberg.de
}

\begin{abstract}
:
There are various applications in high voltage or explosive environments, where sensor powering as well as data transmission has to be electrically isolated. A possible solution can be based on optically powered optical sensor links. These links need typically four different optical components: For the data communication a fast LED as a transmitter and a photo diode as a receiver, furthermore for sensor feeding a high-power light source and a photonic power converter.
\end{abstract}

In this paper it will be demonstrated how to realize an optically powered optical sensor link, using one kind of optical component only, strictly speaking LEDs, for all these tasks.

Key words: remotely powered sensors, optical fibers, galvanic isolation, sensor networks.

\section{Introduction}

A light-emitting diode (LED) can be operated not only as a light source, but always as a photo diode too. Even if a LED is optimized to emit light its basic construction is a semiconductor component with $p-n$ junction like any other photodiodes which are used for receivers in optical data transmissions. Therefore a LED can be used as a receiver for data transmission as well as a converter for remote powering. These properties were already published in 1973 by Forrest W. Mims [1].

But until now the remotely powered sensor systems still uses either one fiber for the power transmission and another one for the data transmission or couples them, using an optical splitter (see Fig. 1).

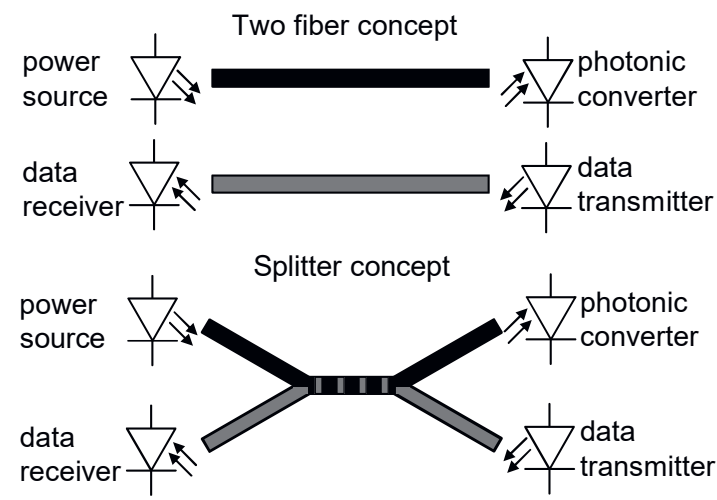

Fig. 1: Current concepts for remotely powered sensor systems
In contrast to this concept, the LED feature described above allows developing of a star topology sensor network, where each node uses a single LED as combined transmitter and photonic power converter. The central node needs one LED per sensor node for remote powering and receiving the measured data. This concept requires a single optical fiber per node only, without optical splitters, (see Fig. 2).

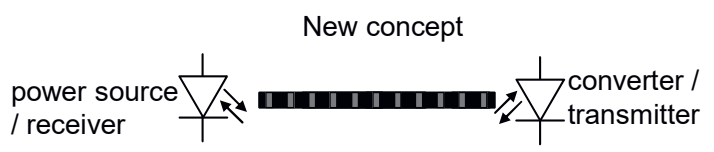

Fig. 2: New concept using one LED as power source and data receiver and another of the same type as power converter and data transmitter

This new concept is realized as a prototype of a sensor network.

\section{Basic System Principle}

The realized sensor network uses polymer optical fibers for power and data transmission and commercial available high-power LED.

A LED is used as a power source and optical data receiver. The same type of LED acts as a photonic power converter and optical data transmitter. Even if this solution does not provide a very high efficiency, it can offer easy mass production and the use of a low number of components. Therefore, it can be employed in low cost applications with limited power requirements. 
Since each sensor node is connected via a single fiber, the transmission of energy and data is implemented by a time division duplex (TDD) procedure (see Fig. 3). To realize this procedure, energy storage at the sensor nodes is necessary to provide power by the time the sensor node send the measurement data, even if the required time for the data transmission is shorter than the available time for powering.

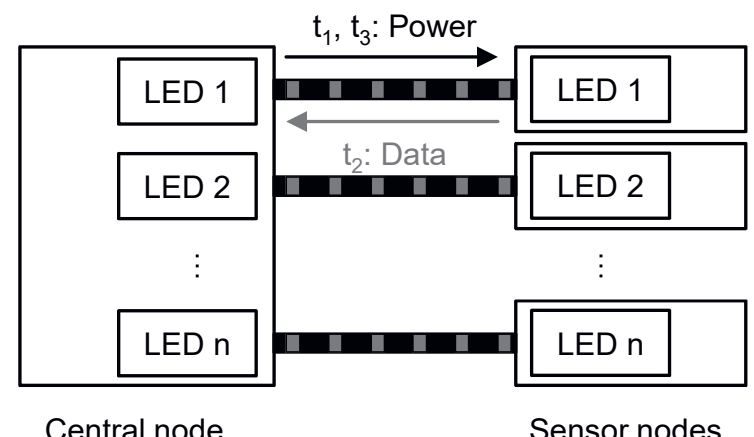

Fig. 3: System concept using TDD for energy and data transmission

The principle of the TDD is based on a periodic cycle. At first the sensor nodes receive energy to charge $\left(t_{1}\right)$. Then the nodes perform measurements and send the data $\left(t_{2}\right)$. Afterwards, the central node powers the sensors again $\left(t_{3}\right)$ (see Fig. 4).

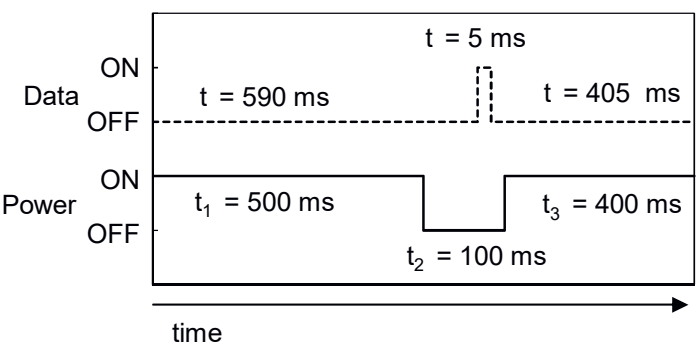

Fig. 4: Timing diagram for the power and data transmission with a period of 1 second.

\section{LED Characteristic}

To realize an efficient system at reasonable costs, characterization and comparison of different LEDs are required.

Depending on the operation mode (as a power source, as a power converter, as a data transmitter or as a data receiver) it is necessary to know various characteristics of the LEDs. For five different LEDs these characteristics are shown below.

These different LEDs are all produced by Cree and are of the same series. The central emitting wavelengths are between $450 \mathrm{~nm}$ (royal blue) and $650 \mathrm{~nm}$ (red).

\section{Coupling efficiency}

To provide as much light as possible to the sensor node, an efficient coupling into the fiber is required. Since a LED has generally a Lambertian radiation pattern and a limited power density, it is necessary to use an optical fiber with a high acceptance angle, which is defined by the numerical aperture (NA), and a large diameter. Employing of an additional coupling optics does not bring a sufficient improvement.

There are different types of optical fibers, like single mode glass fibers (SM), multi-mode glass fibers (MM), hybrid polymer/glass fibers (PCS) and multimode optical polymer fibers (POF) [2].

The polymer optical fiber used in the prototype system has a large diameter and a high NA. These properties outweigh the disadvantages of this fiber, like a higher attenuation in regard to $\mathrm{MM}$ and SM fibers, if typical sensor link lengths up to 10 meters are taken into account. Due to the low bandwidth of the LEDs the limited bandwidth does not play a significant role for this application as well.

For the LED with a Lambertian radiation pattern the coupling efficiency depends on the acceptance angle of the fiber ( $\eta_{\text {angle }}$ ) as follows (1).

$\eta_{\text {angle }}=N A^{2}$

The coupling efficiency depends also on the fiber diameter $\left(\eta_{\text {diameter }}\right)$ In the case that the LED is connected without any distance to the fiber it can be easily calculated as a ratio of the LED area under the fiber end face area.

For example, a LED with a rectangular active area of $1 \mathrm{~mm}^{2}$ has a calculated overall coupling efficiency of about $19 \%\left(\eta_{\text {angle }}=25 \%\right.$, $\eta_{\text {diameter }}$ $=75.4 \%$ ) by using a POF with a diameter of 1 $\mathrm{mm}$ and about $0.01 \%$ only for a multi-mode glass fiber with $50 \mu \mathrm{m}$ diameter $\left(\eta_{\text {angle }}=5 \%\right.$, $\eta_{\text {diameter }}=0.20 \%$ ).

Since the calculation of the coupling efficiency do not include all effects influencing the light, provided to the sensor nodes (i.e. losses due to the end face preparation quality, attenuation losses of the fiber), it is still necessary to verify the calculations by measurements.

In order to calculate the efficiency $(\eta)$ the optical power $\left(P_{N}\right)$ of the LED at the typical current was measured and compared with the fiber coupled optical power $\left(P_{C}\right)$ after one meter (see Tab. 1). 
Tab. 1: Optical power, fiber couple power (into standard POF, $1 \mathrm{~mm}, N A=0.50$ ) and efficiency of the measured LEDs [3]

\begin{tabular}{|c|c|c|c|}
\hline LED & $\mathrm{P}_{\mathrm{N}} / \mathrm{mW}$ & $\mathrm{P}_{\mathrm{C}} / \mathrm{mW}$ & $\eta / \%$ \\
\hline Cree royal blue & 930 & 201 & 21.6 \\
\hline Cree blue & 716 & 130 & 18.2 \\
\hline Cree green & 281 & 55 & 19.3 \\
\hline Cree red-orange & 331 & 57 & 17.1 \\
\hline Cree red & 410 & 83 & 20.2 \\
\hline
\end{tabular}

The measurements show that the coupling efficiency is nearly the same for all LEDs, but due to the higher optical power of the royal blue LED it provides up to four times higher fibercoupled power compared to the other LEDs.

\section{Responsivity}

Another important factor for powering the sensor nodes is the sensitivity of the LEDs to their own emitted light spectrum. This so called mutual responsivity of the LEDs gives an indication of the maximum current available for any connected sensor electronics. To get the mutual responsivity, it is necessary to compare the spectral responsivity $(R(\lambda))$ and the spectrally resolved radiant power $(\Phi(\lambda))$ of the LEDs (see Fig. 5 for an example for the red LED).

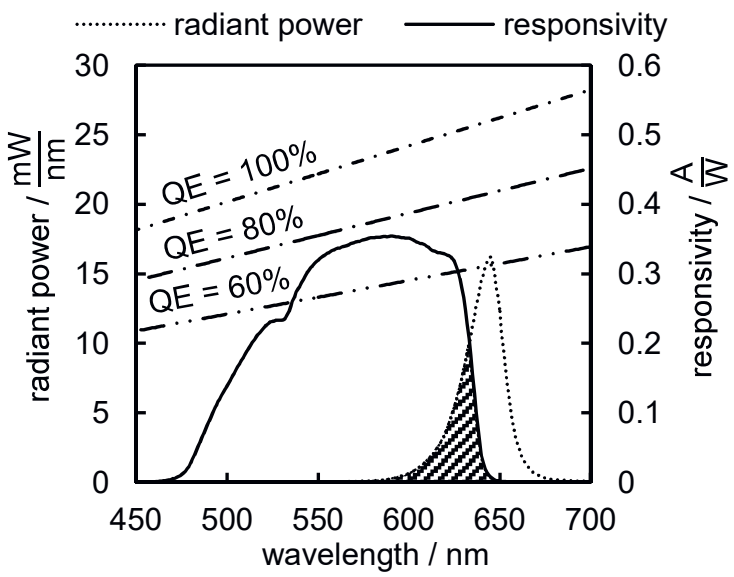

Fig. 5: Spectral responsivity and spectrally resolved radiant power of the red $L E D$ compared to a quantum efficiency of $60 \%, 80 \%$ and $100 \%$. The hatched area represents the range of mutual absorption.

These two curves give a range of mutual absorption of the LED, where photons absorbed and converted into electrons which generate a current.

This measurement indicates two important conclusions: The responsivity of a LED, designed only for light emission, can be surprisingly high. For this sample, the maximum value for the responsivity is about $0.36 \mathrm{~A} / \mathrm{W}$ at $580 \mathrm{~nm}$. This corresponds to a quantum efficiency (QE) of $77 \%$. This is not much worse than the best commercial available converter components [4].

The second result of this measurement is that a significant amount of the LED's emission can be back converted into electrical energy using the same type of LED.

The mutual responsivity $\left(R_{S}\right)$ is a ratio between this mutual absorption and the integrated radiant power (2).

$$
R_{S}=\frac{\int R(\lambda) \cdot \Phi(\lambda) d \lambda}{\int \Phi(\lambda) d \lambda}
$$

The results for the mutual responsivity of the different LEDs are shown in Tab. 2.

Tab. 2: Mutual responsivity of the LEDs under test

\begin{tabular}{|c|c|}
\hline LED & $\mathrm{R}_{\mathrm{S}} / \frac{\mathrm{A}}{\mathrm{W}}$ \\
\hline Cree royal blue & 0.0218 \\
\hline Cree blue & 0.0131 \\
\hline Cree green & 0.0054 \\
\hline Cree red-orange & 0.0897 \\
\hline Cree red & 0.1073 \\
\hline
\end{tabular}

It can be seen, that the mutual responsivity of the red LED is the highest one. It has up to 20 times higher responsivity than other LEDs. Just the red-orange LED provides almost the same value.

\section{Short circuit current and open-circuit voltage}

For the electrical design of the sensor boards as well as for the calculation of the maximum possible power consumption of them, it is necessary to know the LEDs' maximum short circuit current (ISc) and open-circuit voltage $\left(\cup_{\mathrm{OC}}\right)$. These measurements are performed with a complete setup including sending LED, fiber and receiving LED.

A rough estimation of the short circuit current (Est. I IS) can be done by using the mutual responsivity and the fiber coupled power of the LEDs. This estimation does not take into account the coupling efficiency on the receiving LED. Ideally these losses are only reduced to geometrical losses.

For every measurement two LEDs of the same type are connected by a POF with one meter lengths. To achieve the maximum values, the fiber must be aligned to the LEDs. The measurement results show a noticeable 
variation between the theoretical and measured values (see Tab. 3).

Tab. 3: Estimated and measured short circuit current and open-circuit voltage

\begin{tabular}{|c|c|c|c|}
\hline LED & $\begin{array}{c}\text { Est. } \mathrm{I}_{\mathrm{sc}} / \\
\mathrm{mA}\end{array}$ & $\begin{array}{c}\mathrm{I}_{\mathrm{sc}} / \\
\mathrm{mA}\end{array}$ & $\begin{array}{c}\mathrm{U}_{\mathrm{OC}} / \\
\mathrm{V}\end{array}$ \\
\hline Cree royal blue & 4.38 & 2.71 & 2.52 \\
\hline Cree blue & 1.70 & 1.34 & 2.41 \\
\hline Cree green & 0.30 & 0.16 & 2.03 \\
\hline Cree red-orange & 5.11 & 4.48 & 1.72 \\
\hline Cree red & 8.91 & 6.44 & 1.72 \\
\hline
\end{tabular}

Even though the royal blue LED provides the highest fiber coupled power, the red one has a much higher short circuit current, caused by the higher responsivity. Also the higher open circuit voltage of the royal blue LED does not compensate this. Even if it is not possible to determine the exact available electrical power by this measurement, it gives a good indication, since this power corresponds nearly to the product of $90 \%$ of the short circuit current and $90 \%$ of the open circuit voltage. Furthermore this measurement can be implemented easier than a complete I-V characteristic.

The variation between the theoretical and the measured values for the short circuit current are most likely caused by geometrical losses between the fiber and the receiving LED.

\section{Demonstrator}

Using the measurement results for the different LEDs the demonstration setup was designed [5], [7].

The demonstrator consists of a central board and four sensor units.

The LED Cree XQ-E HI red was used, since it provides the highest usable electrical power. The maximum power $\left(\mathrm{P}_{\mathrm{MPP}}\right)$ is defined by the maximum power point (MPP), which was determined by measuring the I-V characteristic of the red LED (see Fig. 6).

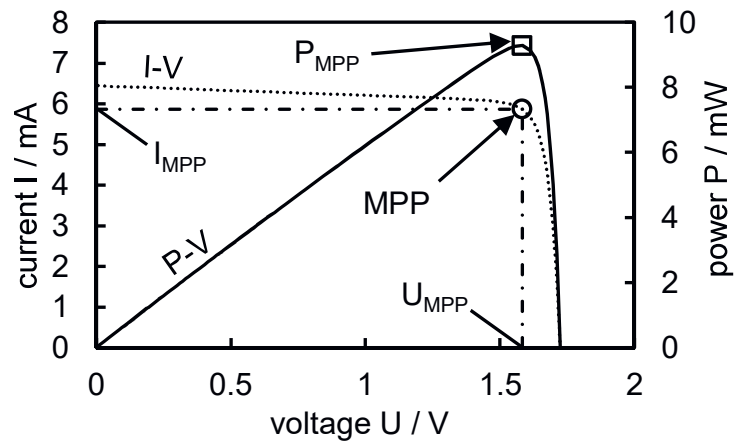

Fig. 6: I-V and P-V characteristic of the Cree XQ-E HI red
During this measurement the current $\left(I_{\mathrm{MPP}}\right)$ and the voltage $\left(\mathrm{U}_{\mathrm{MPP}}\right)$ of the maximum power point were measured as well (see Tab. 4).

Tab. 4: Maximum power, current and voltage for the Cree XQ-E HI red

\begin{tabular}{|c|c|c|c|}
\hline LED name & $\begin{array}{c}\mathrm{P}_{\mathrm{MPP}} / \\
\mathrm{mW}\end{array}$ & $\begin{array}{c}\mathrm{I}_{\mathrm{MPP}} / \\
\mathrm{mA}\end{array}$ & $\begin{array}{c}\mathrm{U}_{\mathrm{MPP}} / \\
\mathrm{V}\end{array}$ \\
\hline Cree $\mathrm{XQ}-\mathrm{E}$ HI red & 9.29 & 5.87 & 1.58 \\
\hline
\end{tabular}

\section{Central board}

The central board powers the connected sensor boards and receives the measurement data from the sensors. The key element is a microcontroller (MCU) "Kinetis MKL05" (ARM Cortex-M0+ based) of NXP, former Freescale.

To power the sensors the microcontroller controls the LED via a LED driver using pulsewidth modulation (PWM).

Receive of the sensor data is realized by calling the sensors one by one, stopping the supply of optical power for a certain time which causes the microcontroller on the sensor boards to perform a measurement.

Then the powering LEDs are used as a receiver for the sensor data, which are realized as universal asynchronous receiver/transmitter (UART). By using an eightfold switch (MUX), the current receiving LED is toggled to an operational amplifier, which output is then connected to the microcontroller. This is necessary since there is only one UART communication interface available on the MKL05.

Finally the data is sent to a connected $\mathrm{PC}$ using an UART/USB converter.

A block diagram of the sensor central board is shown in Fig. 7.

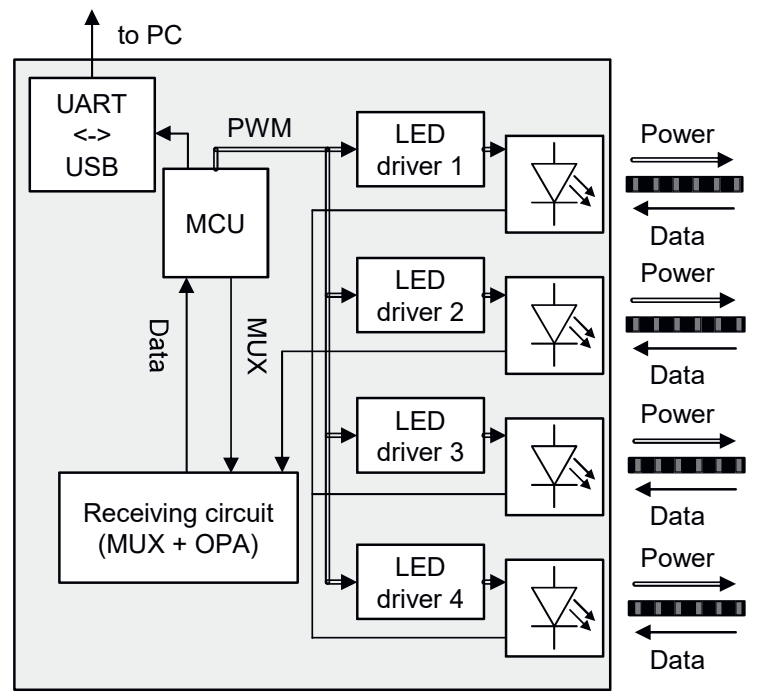

Fig. 7: Block diagram of the sensor central board 
An appropriate printed circuit board was designed and built up to realize the central board in hardware (see Fig. 8).

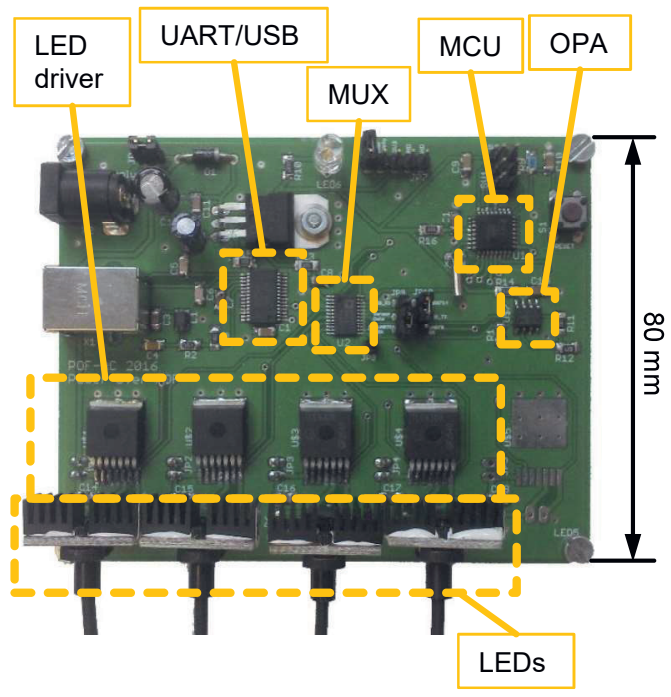

Fig. 8: Central board hardware setup [5]

\section{Sensor unit}

Four sensor units use microcontrollers (MCU) to collect the data from the sensors connected to the boards via $\mathrm{I}^{2} \mathrm{C}$ and then send the data to the central board. The block diagram of the sensor unit is shown in Fig. 9.

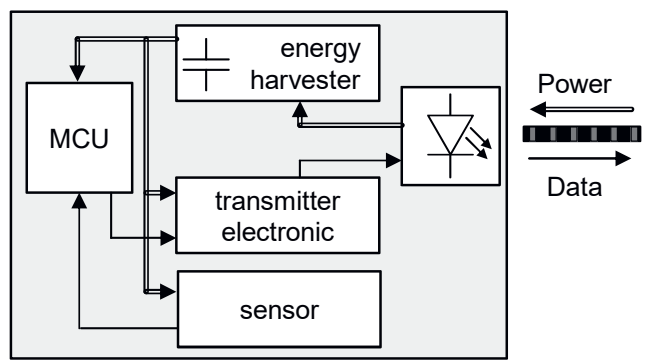

Fig. 9: Block diagram of the sensor unit

To perform a data transmission, the sensor board must be charged by the received optical power. The reason is that while the board sends the sensor data to the central board there is no energy transmission, because a single fiber is used for powering and data transmission.

An energy harvester ("bq25570" from Texas Instruments) is used to charge a $22 \mathrm{mF}$ supercapacitor with the photocurrent generated by the LED. Furthermore this energy harvester provides the supply voltage for all consumers on the board.

This harvester is already equipped with an internal boost charger for charging the capacitor. The maximum charged voltage is 5 Volt, while the integrated and adjustable stepdown converter supplies the load with 3.3 Volt (see Fig. 10).

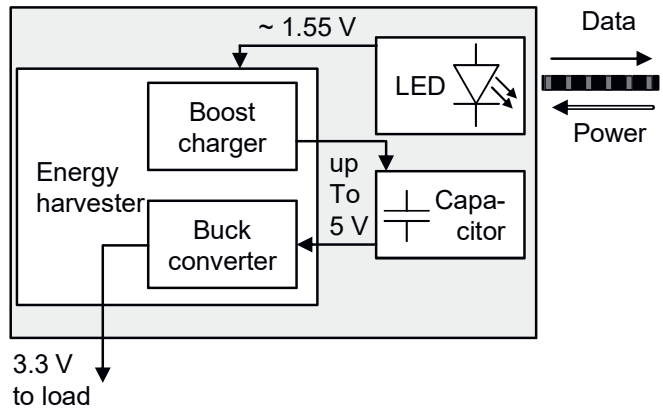

Fig. 10: Block diagram of the energy harvester

The microcontroller is the same as the one on the central board (MKL05). The reasons to employ this MCU are its available different low power modes. As soon as the converter supplies the microcontroller with the required $3.3 \mathrm{~V}$ it boots and then switches into a stop mode (VLPS). For this mode, the current consumption of the controller is only at $2.25 \mu \mathrm{A}$. When the power LED is shut off, the microcontroller recognize an interrupt and switches to a very low power mode, to perform the measurements and send the data to the central board. In this mode the current consumption of the MCU is below $243 \mu \mathrm{A}$. [6]

Since the LED is a power receiver and a data transmitter as well, it is necessary to switch between these operational modes. A field-effect transistor, controlled by the microcontroller, is used to accomplish this. The data transmission is serial, using the UART interface of the microcontroller, with 9600 baud.

Every sensor with $\mathrm{I}^{2} \mathrm{C}$ is suitable for the system. The only limitation is the overall power consumption, required for the microcontroller and the data transmission. It must be lower than the power supplied by the central board. For this demonstrator the following four different sensors were used:

- A temperature sensor (TMP75)

Range: $-40{ }^{\circ} \mathrm{C}-125^{\circ} \mathrm{C}$

Resolution: $0.125^{\circ} \mathrm{C}$

- An atmospheric pressure sensor (MS5637)

Range: $10-2000$ mbar

Resolution: $<0.11 \mathrm{mbar}$

- A relative humidity sensor (SHT21)

Range: 0 - $100 \% \mathrm{RH}$

Resolution: $0.04 \% \mathrm{R}$

- A photoelectric sensor (TCST2103)

Range: Open-Closed

The layout for the sensor boards is the same for all sensor types. Only a small adaptor board has to be changed, depending on the sensor used. As an example the circuit board for the temperature sensor is shown in Fig. 11. 


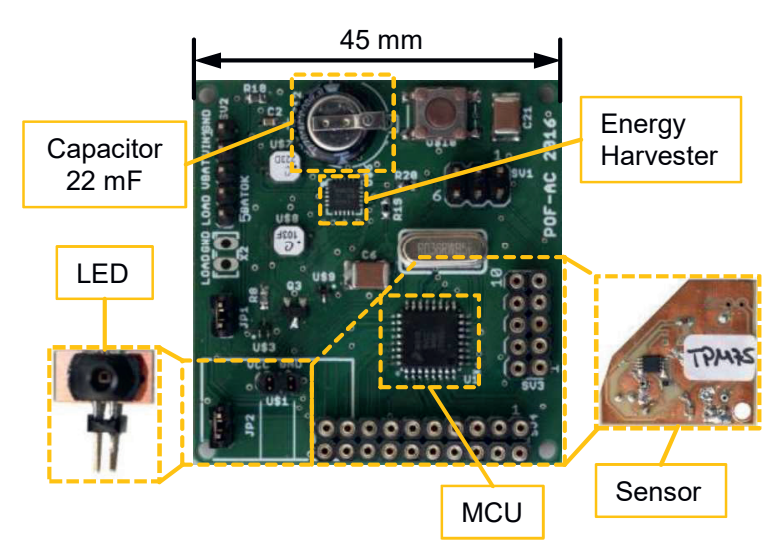

Fig. 11: Sensor board hardware setup (TMP75) [5]

The time until the sensor is ready for measurements, depends on the fiber length which connects the sensor nodes to the central board, and the residual charge in the capacitor. The capacitor keeps a residual charge above 2 Volt for several days.

\section{Demonstrator parameters}

For the above described demonstrator, the following parameters are achieved:

- Measurement cycle: 1 Second/Sensor

- Maximum fiber length: $10 \mathrm{~m}$

- $\quad$ Time until sensor is ready (1m fiber): $131 \mathrm{~s}$ (cold-start operation), $17 \mathrm{~s}$ (capacitor charge: $2 \mathrm{~V}$ )

- Time until cold-start operation necessary: 2-3 days

- $\quad$ Up to 120 Measurements possible without external powering (capacitor charge: 5V)

\section{Outlook and Conclusion}

Optical powering over fibers is a wellestablished technology. The transmission of several watts of electrical power is possible with near infrared laser diodes, glass fibers and optimized converters.

The use of LED in combination with large diameter and NA POF can expand the field of application to low cost and high volumes. A special solution is the use of power LED as transmitters and converters alternatingly. This allows to reduce the number of electrical components and to use a single fiber without splitters. A significant disadvantage of the given approach is the limited mutual responsivity of the LEDs. The use of low power electronics makes this fact acceptable.

In a first demonstration we have successfully proven the operation of a sensor network, equipped with the same type optical semiconductor device (a red power LED). Moreover, we have proven that LEDs with other visible wavelength can also be used as converters and receivers with surprisingly good parameters. Gallium nitride ( $\mathrm{GaN}$ ) based LED may offer advantages in the temperature stability and lifetime [8].

In the next steps we plan to realize sensor links operating at a shorter wavelength. The reduction of the sensor power consumption, increase of the possible data rate and improvements in the energy harvesting process are also under consideration. These investigations are planned in cooperation with Avago Technologies $\mathrm{GmbH}$, A Broadcom Limited Company.

Further improvements can be achieved by using dielectric taper couplers, which has been shown in [9], as well.

\section{Acknowledgment}

Parts of this work have been funded by the Staedler-Stiftung within the project "OSALED Optische Sensor-Anbindung mit Leuchtdioden".

\section{References}

[1] F. M. Mims III, LED Circuits and Projects (1973), Howard W. Sams \& Co., Inc., New York, NY

[2] O. Ziemann, J. Krauser, P. E. Zamzow, W. Daum, POF Handbook: Optical Short Range Transmission Systems - 2nd Edition (2009), Berlin Heidelberg: Springer-Verlag

[3] C. Wächter, Übersicht der Eigenschaften kommerziell verfügbarer High-Power LEDs für Energy Harvesting und optischer Daten- und Energieübertragung (2017), TH Nürnberg Georg Simon Ohm, POF-AC, Nürnberg

[4] Hamamatsu Photonics K.K., S3590-08/-09/-18/19 Si PIN photodiode data sheet (2012), Hamamatsu Photonics K.K., Solid State Division, http://www.hamamatsu.com/resources/pdf/ssd/s3 590-08 etc kpin1052e.pdf

[5] T. Schuster, Entwicklung eines optisch ferngespeisten Sensornetzwerks (2016), TH Nürnberg Georg Simon Ohm, POF-AC, Nürnberg

[6] Freescale Semiconductor, KL05P48M48SF1 Rev 4 data sheet (2014), NXP Semiconductors, http://www.nxp.com/assets/documents/data/en/d ata-sheets/KL05P48M48SF1.pdf

[7] T. Schuster, J. Fischer, O. Ziemann. The use of high power LED as photo converters and optical receivers in low speed POF sensor networks (13.-15.09.2016), Intern. POF Conference POF'2016, Birmingham

[8] J.-M. Wun et al., GaN-based miniaturized cyan light emitting diodes on a patterned sapphire substrate with improved fiber coupling for very high-speed plastic optical fiber communication (Oct. 2012), IEEE Photon Journal, vol. 4, no. 5, pp. 1520-1529

[9] V. Lange, B. Kopf, S. Storm, R. Hönl. Efficient Symmetric Led-POF Coupling (22.-24.09.2015), Intern. POF Conference POF'2015, Nürnberg 